\title{
El desarrollo de la comunicación científica de los estudiantes de la Universidad Tecnológica ECOTEC mediante los avances tecnológicos
}

\section{The development of the scientific communication of the students of the technological university ECOTEC through the technological advances}

\author{
MSc. Nadia Aurora González Rodríguez ${ }^{1}$ \\ ngonzalez@ecotec.edu.ec
}

Recibido: 1/12/2017, Aceptado: 1/02/2018

\begin{abstract}
RESUMEN
La comunicación científica es de vital importancia para el desarrollo intelectual de los futuros profesionales. Los diversos adelantos tecnológicos han facilitado el proceso de enseñanza-aprendizaje, dejando atrás las tendencias antiguas asociadas con la educación superior dando paso a una manera diferente de aprender e investigar. Este artículo tiene como objetivo analizar el desarrollo de la comunicación científica de los estudiantes de la Universidad Tecnológica ECOTEC mediante los avances tecnológicos. Para cumplir con este propósito se puso en práctica la utilización del portafolio de un curso como uno de los recursos tecnológicos asequibles a los estudiantes, lo cual propició un mejor aprovechamiento de las fuentes de información para la adecuada producción científica. En tal sentido, se tuvo en consideración tanto métodos teóricos como empíricos en la consulta de información y en la puesta en práctica del recurso tecnológico seleccionado para el desarrollo de la comunicación científica.
\end{abstract}

Palabras clave: tecnología educativa, enseñanza-aprendizaje, portafolio

\section{ABSTRACT}

Scientific communication is of vital importance for the intellectual development of future professionals. The various technological advances have facilitated the teaching-learning process, leaving of the students of the Technological University ECOTEC through technological advances. To fulfill this purpose, the use of the portfolio of a course as one of the technological resources available to students was put into practice, which led to a better use of information sources for adequate scientific production. In this sense, both theoretical and empirical methods were considered in the consultation of information and in the implementation of the selected technological resource for the development of scientific communication.

\footnotetext{
${ }^{1}$ Máster en Ciencias de la Educación. Docente Titular y Coordinadora de Publicaciones de la Universidad Tecnológica ECOTEC. Ecuador
} 
Keywords: educational technology, teaching-learning, portfolio

\section{Introducción}

La educación superior se ha visto influenciada por cambios significativos en relación con los adelantos tecnológicos. Los avances científicos del siglo XX y del XXI en el ámbito de la informática y las comunicaciones ha propiciado la interacción dinámica aplicada al aprendizaje; donde la información es prácticamente infinita tomando un enfoque más interactivo y competitivo en un mundo globalizado.

La tecnología en el siglo XXI permitió a más personas el acceso a la información, y como se mencionó anteriormente todo está basado en el conocimiento, mientras más mentes tengan acceso a la información se creará una competición mayor entre sociedades y personas, por querer liderar dichas áreas. El conocimiento crea una competición constante, mientras que también abre nuestras mentes. Por tanto, la misión de los docentes como agentes de cambio, debe ser la adecuada utilización de una metodología innovadora acorde a las transformaciones que se han venido produciendo en relación con el uso de la tecnología.

Para el cumplimiento de lo expuesto anteriormente, parte la siguiente investigación donde el objetivo principal es analizar el desarrollo de la comunicación científica de los estudiantes de la Universidad Tecnológica ECOTEC, a través de los avances tecnológicos, como una forma de compartir experiencias productivas en función de la gestión de la calidad del proceso de enseñanza-aprendizaje. Por esta razón, a continuación, se establecen algunos criterios fundamentales para comprender la relevancia de esta investigación y se comparten los resultados obtenidos de la práctica educativa en relación con utilización de la biblioteca virtual y el portafolio estudiantil, para el desarrollo de la comunicación científica.

Tecnología Educacional y su aplicación a la metodología de la enseñanza

La educación en el siglo XX fue una manera de estudio muy centralizada en medios muy limitados como libros, periódicos, televisión; puesto que durante este siglo no se socializaba la información de la manera que actualmente se hace en este mundo globalizado, siendo así más difícil de encontrar información pertinente. De esta manera, uno las fuentes primarias de información durante gran parte del siglo XX ha sido el libro, su invención permitió a la humanidad poder impregnar y conservar eventos históricos, datos científicos y cualquier tipo de información.

Sin embargo, a finales del siglo XX se comenzó la Revolución Tecnológica, su implementación se pudo evidenciar en el ámbito de la educación; aunque solo en algunos países del mundo. Por cuanto, no se puede identificar a esta época como un siglo tecnológico, ya que la inmensa mayoría de la población mundial no contaba con el acceso al uso de tecnología; sin embargo, el siglo XX se puede considerar como la base para la era tecnológica.

En el siglo XXI la educación cambia su perspectiva, ya no solo tiene un enfoque de crecimiento intelectual, ni social, ahora se relaciona con el crecimiento económico, empresarial, siendo la educación y el conocimiento la base de lo que actualmente maneja las economías y sociedades del gran parte del mundo. Toffler, A. \& Toffler, H. (1996, p.21) afirman: "Todos los sistemas económicos descansan sobre una base de conocimientos. Todas las empresas dependen de la existencia previa de este recurso de construcción social". 


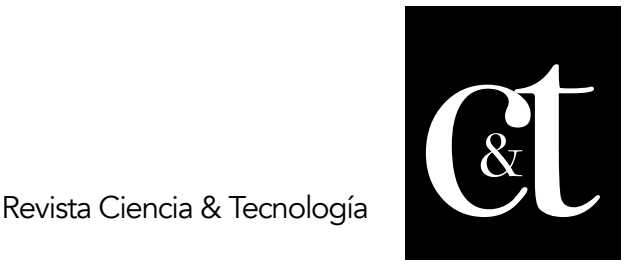

No. 18, 30 de abril de 2018

ISSN impreso: 1390 - 6321

La tecnología educativa es la combinación entre la enseñanza e innovaciones tecnológicas, capaces de potencializar ciertos aspectos del aprendizaje, un ejemplo preciso es Internet que se acopló a la educación con el fin de facilitar la búsqueda de información deseada, a diferencia del siglo pasado donde su fuente principal eran los libros. Internet, además de ser una herramienta que ayuda a la adquisición de conocimientos, se ha convertido en facilitadora de oportunidades. La globalización y la tecnología han permitido el acceso a la educación a una gran cantidad de personas, ya sea dentro o fuera de su país natal (Olguín, 2012).

La tecnología educativa es el resultado de las aplicaciones de diferentes concepciones y teorías educativas para la resolución de un amplio espectro de problemas y situaciones referidos a la enseñanza y el aprendizaje; apoyadas en las Tecnologías de Información y Comunicación (p. 2).

Internet es claramente uno de los avances más relevantes perteneciente a la tecnología educativa, como manifiestan Majó y Marqués (2002).

De los grandes inventos y descubrimientos de las últimas décadas, ni la radiotelevisión, ni la energía nuclear, ni la conquista del espacio, ni los ordenadores...quizás ninguno ha ejercido un papel tan decisivo en la evolución ( $y$ a veces revolución) de nuestra sociedad como Internet (p. 2).

La aplicación de la tecnología en la educación como se ha mencionado tiene una amplia gama de beneficios, las invenciones que se han dado en la era tecnológica se han ido acoplando oportunamente a la metodología de la enseñanza de cada maestro, algunas de las invenciones y sus aplicaciones más comunes son:

Dispositivos audiovisuales: Son capaces de reproducir videos y sonidos, obteniendo así un enfoque más didáctico en cuanto al contenido que necesite una representación gráfica. Dispositivos capaces de realizar dicha acción. Tales como: proyectores, computadoras, teléfonos celulares, tabletas (computadora).

Internet: actualmente como fuente principal de información, donde se pueden encontrar desde libros muy antiguos a transmisiones en vivo. Unas de las principales búsquedas educacionales en internet son: Libros, revistas, etc., videos sobre hechos históricos, videos sobre hechos actuales o pasados, artículos de opinión, revistas, blogs de discusión. Dispositivos de almacenamiento: Dispositivos donde se pueden llevar archivos que contengan información de relevancia académica, así logrando aminorar la carga de grandes libros, tareas o material de apoyo, también se ayuda al no uso de papel. Tecnología óptica (CD, DVD, Blu-ray, etc.), Tecnología Electrónica: Pendrives, Tarjeta de memoria, etc.

Portafolio: el portafolio se fundamenta en la idea de que la evaluación muestra la manera en cómo una persona que aprende organiza su aprendizaje, o sea, testimonia de alguna manera su estilo para aprender (Murillo, 2012, p.3).

La implementación de la tecnología en las universidades ecuatorianas ha sido un avance importante, puesto que los adelantos tecnológicos remplazaron los antiguos procesos asociados con la manera de enseñar. Los métodos de investigación para la entrega de deberes, la comprobación de la originalidad mediante el sistema de anti- 


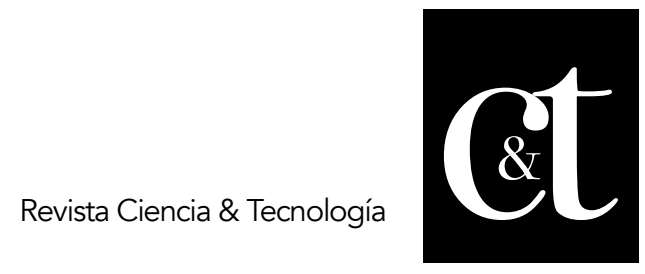

No. 18, 30 de abril de 2018

ISSN impreso: 1390 - 6321

plagio, lo que propició el aumento del rendimiento académico y el nivel de eficiencia en la enseñanza. "Competitividad implica conocimiento, tecnología, manejo de información, destrezas, significa elevar la calidad de nuestros sistemas educativos, ponerlos al nivel de sus similares internacionales, flexibilizar los sistemas de reconocimiento, armonización de estudios y movilización de profesionales, docentes y estudiantes" (Baquero, 2004).

En el caso de Ecuador se ha implementado algunas de estas tecnologías educacionales, para potencializar el desarrollo social y cognitivo de los estudiantes, logrando de esta manera facilitar oportunidades y decisiones entorno al estudio. Una de las gestiones realizadas por el gobierno ecuatoriano se relaciona a decir de Ramírez (2006, p. 69) con un Programa Nacional de Tele-educación, el cual está impulsado por el Ministerio de Educación y Cultura junto con el Consejo Nacional de Telecomunicaciones y varias universidades.

Sus objetivos son mejorar la calidad de la educación mediante la incorporación de proyectos de tele-educación; desarrollar una infraestructura de telecomunicaciones; desarrollar contenidos nacionales y culturales para Internet; y poner especial atención en la utilización de las tecnologías informáticas como herramientas para promover el desarrollo de habilidades superiores de pensamiento (PNUD, 2001).

Por lo tanto, se evidencia la intención del sistema en implementar políticas que contribuyan a la gestión de la educación mediante las ventajas que aportan el uso de las tecnologías como recursos invaluables de apoyo para el desarrollo de la enseñanza- aprendizaje.

La importancia de la comunicación científica en el desarrollo profesional

La comunicación científica es una manera interesante de establecer intercambios de experiencias en las diferentes áreas de formación. Socializar las investigaciones con otros profesionales refuerza los aprendizajes y establece nuevas maneras de solucionar problemas, según las experiencias desde los diferentes contextos situacionales. "La posibilidad de que los científicos puedan comunicarse entre continentes ha propiciado un aumento de la colaboración en los esfuerzos de investigación y de la labor académica a nivel global, con una mayor movilidad de investigadores y académicos" (Arévalo, 2005, p. 2).

En tal sentido, la comunicación científica es esencial a la naturaleza y práctica de la ciencia, y está presente en todas las etapas del proceso de investigación. Lo cual se ha definido como "el estudio de cómo los investigadores de cualquier campo utilizan y difunden información a través de canales formales e informales" (Borgman, 1989).

Mediante su práctica se desarrollan estrategias de pensamiento que posibilitan el análisis de los fenómenos asociados con dos formas importantes del pensamiento: el análisis crítico y la producción del conocimiento. Esta estrategia resulta de vital importancia en el ámbito de la enseñanza superior y en estos momentos donde la tecnología favorece la producción científica, es el camino más apropiado para establecer las diversas formas de contribuir con la misión social de todo profesional: compartir sus conocimientos con otros.

La capacidad de comunicar efectivamente, es una competencia que aprovecha los conocimientos de un individuo sobre la lengua, así como las prácticas de las TI 
(Tecnologías de la Información), las habilidades y actitudes hacia las personas con quienes él o ella se está comunicando (Rychen y Salganik, 2003 en Romero y Turpo, 2006, p.2).

Los paradigmas de la comunicación han ido cambiando con el tiempo, lo que antes era solo monopolio de uno, ahora es compartido por todos y una de las formas que ha propiciado este fin es el acceso a la tecnología. En estos momentos, tanto la tecnología como la comunicación han estrechado las posibilidades de interrelaciones científicas.

Este tipo de fenómenos están influyendo en la estructura general del sistema de comunicación científica, transformando las funciones y los papeles de diferentes actores. Por esto, los modelos tradicionales de edición y comunicación académica están sujetos permanentemente a nuevos análisis en el contexto del panorama actual definido por las tecnologías de la información (Arévalo, 2005, p. 3).

El profesional de hoy necesita entender la importancia de comunicarse con otros pares, por lo que los futuros profesionales que hoy deben formarse en las universidades deben comprender la necesidad de establecer este tipo de intercambios, que a nivel mundial son parte del diario actuar.

Desarrollo de la comunicación científica mediante el portafolio de un curso

En la actualidad, los profesionales deben involucrarse en la sociedad del conocimiento para que puedan gestionar la información de una manera transversal y con un enfoque interdisciplinario. En el ámbito educativo de la enseñanza superior, la comunicación científica cobra un importante valor, puesto que los enfoques actuales están vinculados con la práctica de escritura de ensayos académicos y artículos científicos, que promueve la comprensión lectora con un análisis crítico sobre las problemáticas relacionadas con su perfil profesional. Para que puedan realizar una eficiente producción científica deben recurrir a diversas fuentes, mediante la búsqueda bibliográfica en revistas electrónicas, que pueden encontrarse en las diferentes bases de datos con acceso a Internet.

Mediante el portafolio para un curso se puede controlar la realización de las investigaciones; aunque a decir de Murillo (2012) existen diferentes tipos como los portafolios de habilidades, portafolio para desarrollar los curriculum personales o historias de vida, portafolio tipo vitrina; el que se utilizará es el portafolio de un curso: Las secciones del portafolio se delimitarán, por ejemplo, según la propuesta de desarrollo del curso, de acuerdo con los temas a tratar; la organización puede estar dada por el docente o realizada por el mismo estudiante. El apoyo, en término de seguimiento, constituye un elemento importante en este tipo de portafolios (Murillo, 2012, p.4).

Mediante la materia de Lenguaje y Comunicación II que se imparte a los estudiantes de las diferentes facultades de la Universidad Tecnológica ECOTEC, se potencia la comunicación científica para la profundización del conocimiento y la resolución práctica de los problemas que se presentan en los diferentes perfiles profesionales del estudiantado. Los estudiantes durante un semestre de clase (4 meses) de la asignatura se involucran en investigaciones conformando un grupo interdisciplinario de tres estudiantes que cursan carreras diferentes. De esta forma, a través de la investigación identifican un problema asociado con su futura profesión y con una 


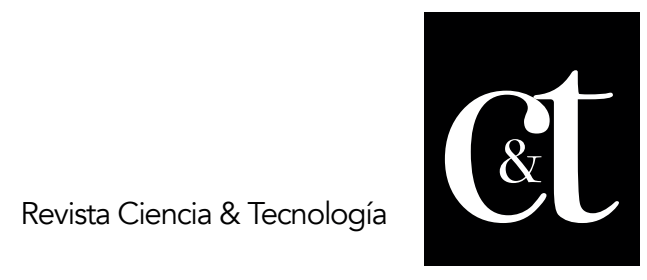

No. 18, 30 de abril de 2018

ISSN impreso: 1390 - 6321

evidente repercusión social, así gestionan una vía de solución donde deben intervenir los diferentes conocimientos de los perfiles profesionales involucrados. Para cumplir con este proceso de investigación, se comienza con entregas parciales de los proyectos.

Los estudiantes responsables de los grupos van colgando en los portafolios los avances de sus investigaciones, al cual tiene acceso el docente y por este medio electrónico se hacen las sugerencias de rectificaciones, para que mejoren el proceso de desarrollo de sus proyectos. Los estudiantes tienen una rúbrica de cumplimiento de los parámetros básicos de acuerdo a los principios fundamentales de la comunicación científica. Una de las condiciones que deben cumplir es la búsqueda de información en la biblioteca virtual que incluyen bases de datos reconocidas, para evitar el uso indiscriminado de fuentes no válidas para la calidad de sus investigaciones. En la entrega previa a la final, las investigaciones son enviadas al sistema de anti plagio para validar la autenticidad de sus producciones científicas.

\section{Metodología}

Los métodos teóricos como el análisis-síntesis y la inducción-deducción que se utilizó en la consulta bibliográfica para la recolección de información. También, se tuvo en consideración la inducción-deducción que se empleó en las encuestas realizadas a los estudiantes que cursaron el semestre para constatar si la aplicación de los recursos tecnológicos propició el desarrollo de sus competencias científicas para su futuro desempeño como profesional.

De los métodos empíricos se empleó el experimento una vez que los estudiantes aplicaron los recursos tecnológicos en función de la puesta en práctica de los aprendizajes obtenidos durante el curso y que se evidencia en la redacción de un artículo científico, como proyecto final del curso, donde deben demostrar el desarrollo de sus competencias. En la evaluación final se demostró que los estudiantes pudieron utilizar los recursos tecnológicos disponibles con efectividad, puesto que se evidenció en los resultados de las calificaciones finales de los estudiantes que participaron en el curso.

Al inicio del curso se realiza un diagnóstico inicial mediante la elaboración de un ensayo académico para comprobar si los estudiantes poseen las competencias comunicativas básicas, que debieron adquirir en la materia de Lenguaje y Comunicación I. En tal sentido, se pudo comprobar que existían algunas falencias desde el punto de vista de la metodología de la investigación y en relación con la escritura académica.

Tomando en consideración estas deficiencias, se comenzó con el proceso de aplicación del portafolio en función de corregir las dificultades individuales en el aprendizaje de los estudiantes. Este recurso demostró, mediante las calificaciones finales de los estudiantes que la progresión del aprendizaje es paulatina, con una adecuada interactividad y control de parte del docente con la debida organización de los tiempos de evaluación para la retroalimentación oportuna de la producción científica. 


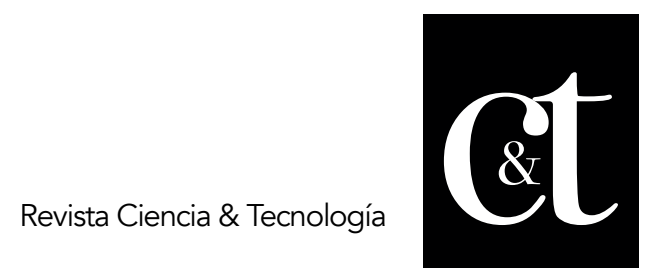

No. 18, 30 de abril de 2018

ISSN impreso: 1390 - 6321

\section{Conclusiones}

El acceso a nuevas fuentes de información brinda una ampliación de conocimiento, por ende, generan una competitividad más grande en el ámbito social, personal, y laboral. La adaptación de nuevos dispositivos dentro y fuera del aula de clase influye en la forma de planificación de las clases de los docentes, para la implementación de nuevas estrategias de enseñanza.

La implementación de los recursos tecnológicos, tal como el portafolio, propicia que la información recopilada por los estudiantes sea más asequible para la rectificación de los errores y el desarrollo de sus competencias científicas. Todo ello, beneficiarán a futuro las estructuras económicas y sociales del país que siempre tendrán como base el conocimiento.

\section{Referencias bibliográficas}

Arévalo, J. A. (2005). Comunicación científica y edición alternativa. Visibilidad y fuentes de información en ByD. Curso: Fuentes de informaciones especializadas y nuevas formas de comunicación científica. $1^{a}$ edición: Salamanca: España. Recuperado de http://eprints.rclis.org/6855/1/Curso_Fuentes1.pdf

Baquero, R. (2004). Analizando unidades de análisis. Los enfoques socio-culturales y el abordaje del desarrollo y el aprendizaje escolar. En Castorina, José Antonio y Dubrovsky, Silvia (comps.), Psicología, cultura y educación. Perspectivas desde la obra de Vigotsky. Buenos Aires: Ediciones Novedades Educativas.

Borgman, C.L. (1989). Bibliometrics and scholarly communication. Communication Research 16 (5):583-599.

Chirino, M. V. (2012). Didáctica de la formación inicial investigativa en las universidades de ciencias pedagógicas. Revista Científico-Metodológica. Juliodiciembre, No. 55, pp.18-24. Recuperado de www.redalyc.org/pdf/3606/360633907004.pdf

Escudero, F. (2013). Redes Sociales. Recuperado de About.com: http://redessociales.about.com/od/comousarlinkedin/a/Que-Es-Linkedin.htm

Majó, J. y Marqués, P. (2002). La revolución educativa en la era Internet. Barcelona, Cisspraxix. Recuperado de http://www.redalyc.org/articulo. oa?id=15801955

Murillo, G. (2012). El portafolio como instrumento clave para la evaluación en educación superior. Revista Actualidades investigativas en educación. Vol. 12, No. 1, pp.1-23. Universidad de Costa Rica.

Olguín, E. (2012). Generalidades de la Tecnología Educativa. Tesis de Maestría de la Universidad Autónoma del Estado de Hidalgo, México: UAEH. Consultado el 31 de julio del 2016.2 Recuperado de: http://cvonline.uaeh.edu.mx/Cursos/Maestria/MTE/Gen03/Tec_educativa/Unida d\%201/ GeneralidadesTecnologiaEducativa.pdf

PNUD (2001). Informe sobre Desarrollo Humano Ecuador 2001. Quito. Recuperado de http://hdr.undp.org/sites/default/files/ecuador_2001_sp.pdf

Ramírez, J.L. (2006). Las tecnologías de la Información y de la Educación en cuatro países latinoamericanos. Red de Revistas Científicas de América Latina y el Caribe, España y Portugal, Vol. 11, Núm. 28, pp. 61-90. Recuperado de http://www.redalyc.org/html/140/14002805/

Romero, M. y Turpo, O. (2015). Serious Games para el desarrollo de las competencias 


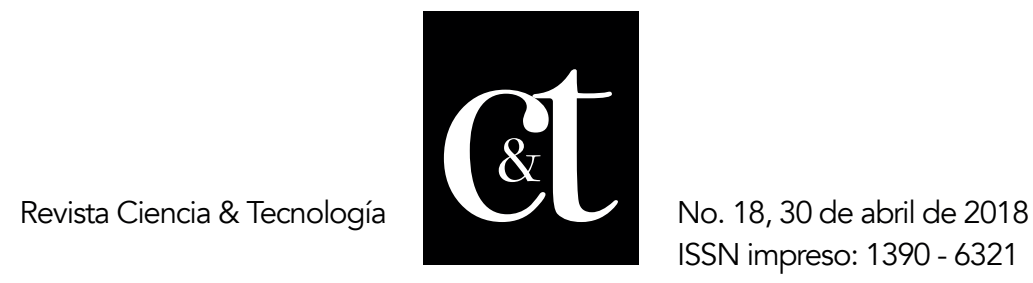

del siglo XXI. Revista de educación a distancia (RED), 34. Consultado el 15 de enero de 2018. Recuperado de http://revistas.um.es/red/article/view/233511

Rychen, D. S., \& Salganik, L. H. Eds. (2003). Key competencies for a successful life and a well-functioning society. Hogrefe \& Huber Publishers.

Toffler, A. \& Toffler, H. (1996). La creación de una nueva civilización. Barcelona: Plaza \& Janés. Recuperado de http://biblio3.url.edu.gt/Libros/2011/La_creac.pdf 